\title{
Transpedicular fixation of the lumbar and lumbosacral spine with screws. Application of the Diapason System
}

\author{
M Yanase, T Sakou, E Taketomi and K Yone \\ Department of Orthopaedic Surgery, Faculty of Medicine, Kagoshima University, 8-35-1 Sakuragaoka, \\ Kagoshima City 891-01, Japan
}

\begin{abstract}
Forty-six patients with the Diapason transpedicular screw system for lumbar and lumbosacral fusions were analysed clinically. No implant breakage was observed, but one rod migrated. Additional sacral screws different in the length of the threaded portion are necessary in this system. Metallosis was noted in a few cases, but posed no significant clinical problems. This system, which is simple in structure, easy to use, and applicable to magnetic resonance scanning after surgery, is considered to be a useful instrumentation.
\end{abstract}

Keywords: transpedicular screw fixation; Diapason system; lumbar spine; lumbosacral spine; posterolateral fusion

\section{Introduction}

In recent years, lumbar and lumbosacral fixation with transpedicular screw is frequently employed in the treatment of degenerative and traumatic conditions of the lumbar spine. Various types of transpedicular screw system have been developed. Among them, the Diapason system (Stryker Company 2725, Michigan, USA) has a quite simple structure made of non-magnetic titanium applicable to magnetic resonance (MR) scanning after surgery. The Diapason system was introduced at our institution in 1992. The present study was undertaken to review the clinical cases who were treated with the Diapason system for the lumbar and lumbosacral stabilisation.

\section{Materials and methods}

Forty-six patients with lumbar disorders treated by transpedicular screw fixation using the Diapason system at our institution were studied. There were 36 men and 10 women, with a mean age of 43.3 years (range 12-74). The diagnosis at the time of surgery included lumbar canal stenosis with instability (12); lumbar disc herniation with instability (9); spondylolytic-listhesis (8); spondylolysis (5); failed back syndrome (9); reconstruction after tumour resection (2); and lumbar congenital scoliosis (1). Patients with traumatic conditions were excluded. Mean follow-up period was 21 months. Numbers of fused motion segments were as follows; one in 23 patients, two in 20 and three in three. Sacral screwing was done in 17.

Operative technique consisted of decompression and discectomy when indicated. The lateral fusion area was then decorticated and packed and the iliac crest bone harvested through the same incision with subcutaneous dissection. Before transpedicular screw insertion, proper posterolateral fusion was done. The site of screw insertion was described by Steffee as the junction of the transverse process and the inferolateral border of the superior articular facet. Before inserting the screws, a small hole was made in the cortex at the point of insertion with a high-speed burr. The pedicle probe was then used to establish the pedicle tract by guiding the tip of the probe along the inner wall of the pedicle into the vertebral body. Intraoperative lateral and anteroposterior radiographs were taken to confirm the proper direction using radiographic markers in the pedicle tract. The screw was then placed without tapping. The smooth surface rods were contoured, positioned, and secured with the olives and blockers.

Clinical outcome and radiographic evaluation were made with regard to complication, breakage and migration of the instruments, demonstrable fusion rate, the appearance of radiolucent zones around the threaded portion of the screws, the state of penetration of the sacral screw through the anterior sacral cortex, and findings on removal of the instruments.

\section{Results}

Wound infection occurred in two $(4.4 \%)$ patients, one superficial and one deep infection. All these infections were healed without removing the instruments. There were no cases of device-related neurovascular injury.

No implant breakage was observed by radiogram or by direct examination when we removed the implant after solid fusion was obtained. Only in one early case, a distal displacement of one rod was observed 2 months after surgery (Figure 1). In this case, tightening the stopcock between the screw and rod was insufficient, and the condition was judged to have been due to our technical failure.

Twenty-six of $31(83.9 \%)$ patients who had been treated 12 months after surgery had successful fusion 
$\mathbf{a}$
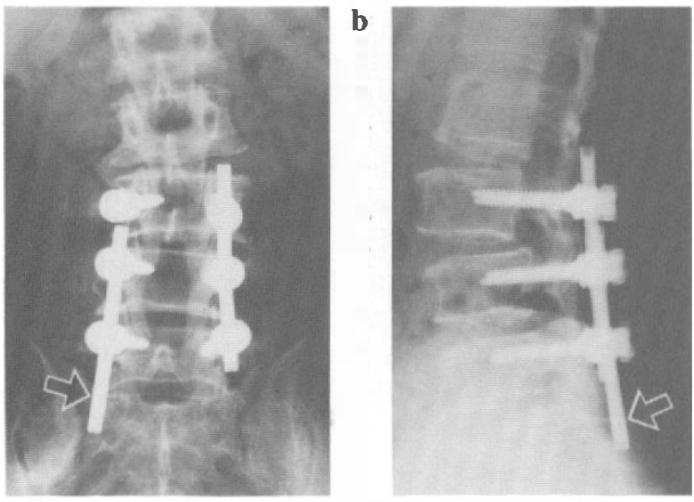

Figure 1 46-year-old man, lumbar canal stenosis with instability. The right rod was displaced distally 2 months after operation.

visualised radiographically. No patient developed obvious pseudoarthrosis that was symptomatic. Radiolucent zones around the threaded portion of the screws were found in six (13.0\%) patients; four of L5 screws, one of L4 screw, and one of sacral screw (Figure 2). In the patient who was observed to have radiolucent zones around the threaded portion of the sacral screws continuous posterolateral bony union was poor.
The sacral screw completely penetrated the anterior cortex of the sacrum in nine $(56.3 \%)$ of 17 patients who underwent sacral screw placement (Figure 3). However none of these cases exhibited symptoms clearly suggestive of neural, vascular or visceral damages.

Metallosis was noted around the instrument in two cases $(13.3 \%)$ of 15 patients who underwent removal of the instruments after solid fusion was obtained (Figure 4). These patients had discomfort in the lumbar region before removal of the instruments.

\section{Discussion}

Transpedicular screw fixation has been widely applied for spinal reconstruction because of its excellent fixation and fusion rate. According to this fact, various transpedicular screw systems have been developed and applied. Breakage of screws has often been reported in rigid type systems, ie plate and screw type. The frequency of implant breakage has been reduced by improvements in the implant through biodynamic studies, but instances of implant breakage are still not infrequent. ${ }^{1}$ The Diapason system is a semirigid instrument composed of screw and smooth rod. In this system, the joint between the screw and the rod has a washer, as a ball-joint, and permits movements of the a

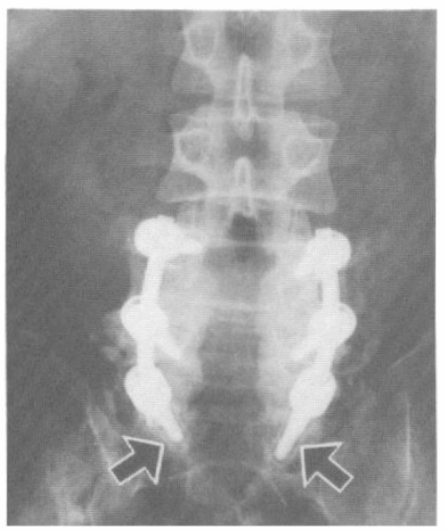

b

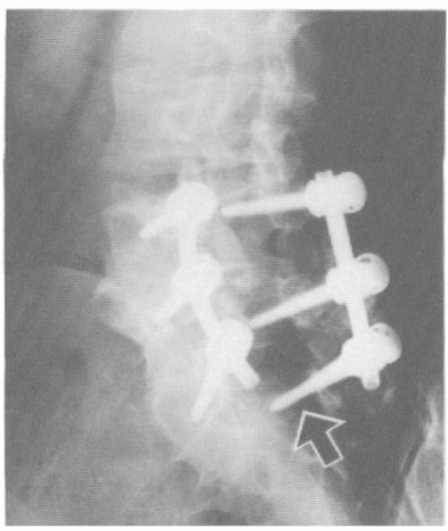

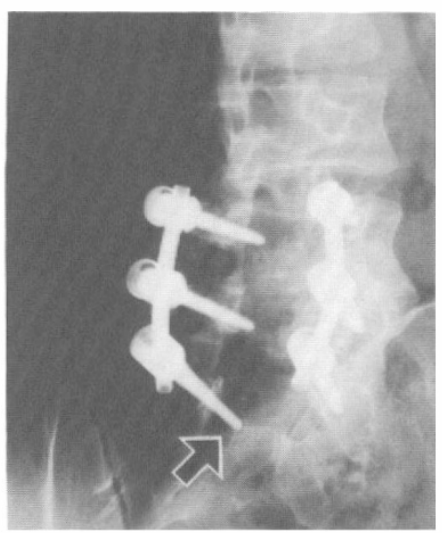

Figure 2 39-year-old man, L4 and 5 spondylolysis. Radiolucent zones of the bilateral sacral screw were found 3 months after surgery.

a

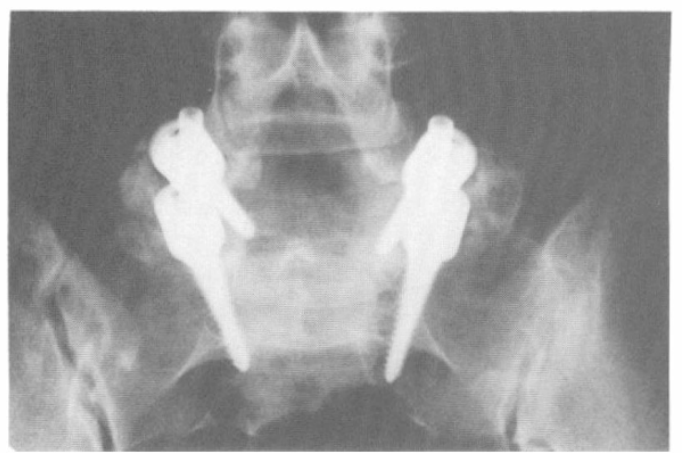

b

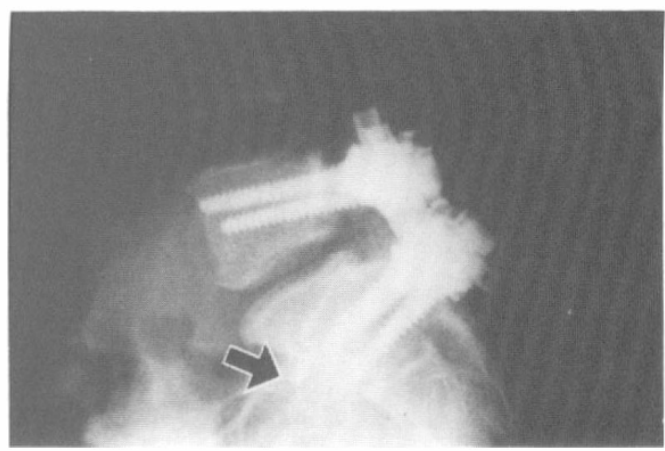

Figure 3 38-year-old female, L5 spondylolytic-listhesis. Note the sacral screw that completely penetrated the anterior cortex of the sacrum. 


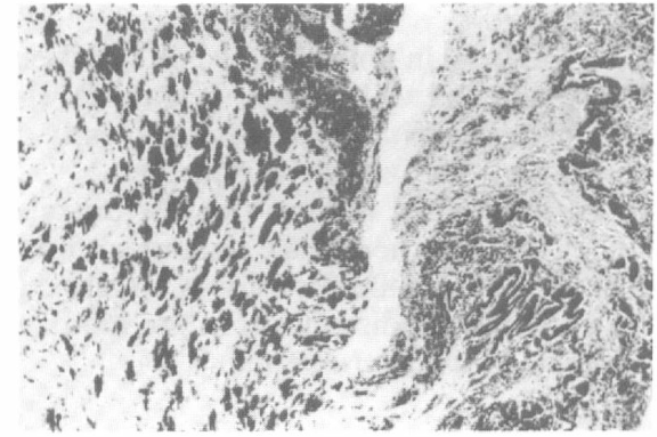

Figure 4 22-year-old man, L5 spondylolysis. (H. E. stain) Metallosis was obvious around the instrument when removed.

screw within a vertical range of $30^{\circ}$ to avoid concentration of mechanical stress. Also, the system is made of a titanium alloy with a modulus of elasticity resembling that of bone. The possibility of implant breakage is expected to be reduced by these modifications, and practically no breakage was observed in our present clinical applied cases.

However, the stopcock between the screw and rod should be tightened with a sufficient torque in order to avoid displacement of the rod as indicated by $\mathrm{Dr}$ Lapresle, who developed the system.

The results of bone union after fixation with the Diapason system were comparable to those after fixation with other pedicular screw systems. ${ }^{2}$ Thus this system is considered to have a sufficient significance as an internal fixation material.

Some investigators reported that radiolucent zones around the threaded portion of both screws at the same segment indicate non-union. ${ }^{3}$ There is a possibility that these images indicate the loosening of the screw, which leads to the loss of adequate fixation. According to our results, however, radiolucent zones were noted around both the screws at the same segment in only one case, and bone union was achieved in this case. Whether radiolucent zones around the threaded portion of both screws at the same segment should be included in evaluation criteria for bone union or not is considered to need further evaluation.

To enhance the strength of lumbosacral fixation, the sacral screw is required to penetrate the anterior cortex of the sacrum. ${ }^{4}$ In this system, the sacral screw is only $40 \mathrm{~mm}$ long. So we achieved bicortical purchase of the sacrum only in $56.3 \%$ of the present cases. Furthermore, to minimise the length of the screw that protrudes beyond the anterior cortex of the sacrum and may cause risks to the anterior sacral structures, ${ }^{5}$ variable lengths of the sacral screw are required.

The macroscopical or microscopical findings that metal wear debris deposits in the soft tissues is generally called metallosis. Fibrous proliferation or focal necrosis of the soft tissues frequently accompanies this state. Titanium, of which this system is made, has an excellent biocompatibility but is not resistant to wear, and there have been a few reports of metallosis. Metallosis was noted in two of our cases, but its clinical symptoms were only lumbar regional discomfort, and no toxic reactions were observed. In our experience of metallosis, we think that removal of the device is favourable after solid union has been achieved.

In the clinical case of multisegmental fixation of the spine with rigid plate and screw type (ie the Steffee system), it is difficult to connect the screw and plate if the screws do not align in the same plane. On the other hand, even if the screws do not align in the same plane, the smooth rod can easily connect the open site of the tulip-type screw of the Diapason system. This sytem is made of non-magnetic titanium applicable to MR scanning and we can obtain good MR images with very low metal artefacts after surgery. In the cases of new lesions such as spinal metastatic tumour appearing in the spinal cord after surgery, this system possesses the advantage of obtaining good MR images which are worth diagnostic evaluation.

\section{References}

1 Davne SH. Complication of lumbar spinal fusion with transpedicular instrumentation. Spine 1992, 17: 184-189.

2 Millan MM. Lumbar and lumbosacral fusions using CotrelDubousset pedicle screws and rods. Spine 1994, 19: 430-434.

3 Hirabayashi S. New criteria of bony union in lumbar posterolateral using pedicle screws. Seikeigeka 1993, 44: 637-942.

4 Zindrick MR. A biomechanical study of intrapedicular screw fixation in the lumbosacral spine. Clin Orthop 1986, 203: 99-112.

5 Licht NJ. Pitfalls of pedicle screw fixation in the sacrum. A cadaver model. Spine 1991, 17: 892-896. 